\title{
CONHECIMENTOS DA EQUIPE ESCOLAR SOBRE DIABETES MELLITUS TIPO 1
}

\author{
CONOCIMIENTO DEL EQUIPO ESCOLAR SOBRE LA DIABETES MELLITUS TIPO \\ 1
}

SCHOOL TEAM KNOWLEDGE ON TYPE 1 DIABETES MELLITUS

\author{
Linamary Chiti CAMARGO ${ }^{1}$ \\ Dariel de CARVALHO ${ }^{2}$
}

\begin{abstract}
RESUMO: Estudo seccional, descritivo, com abordagem qualitativa que objetivou verificar os conhecimentos da equipe escolar de uma escola municipal de Ensino Fundamental I localizado na cidade de Bauru, no interior do estado de São Paulo. A Escola foi selecionada segundo critério de ter pelo menos 1 aluno com diabetes mellitus tipo 1 (DM1) matriculado em sua unidade escolar. Foi aplicado um questionário contendo 19 questões abertas e fechadas contemplando o perfil do grupo e os conhecimentos acerca da doença. Os resultados evidenciaram que $11 \%$ definiram corretamente a doença, $79 \%$ a relacionaram pela ausência da produção de insulina, $11 \%$ afirmaram que a monitorização da glicemia na escola deveria ser realizada de maneira frequente e $58 \%$ responderam que não conviveram com crianças DM1 no ambiente escolar. Com a realização do estudo pode-se concluir que é indispensável a educação da equipe escolar no que se refere ao conhecimento e capacitação sobre DM1, cujos esforços conjuntos, culminem na manutenção da qualidade de vida da criança matriculada na referida unidade escolar.
\end{abstract}

PALAVRAS-CHAVE: Diabetes mellitus tipo 1. Diabetes em escolares. Escola e saúde.

RESUMEN: Se trata de un estudio transversal y descriptivo con un enfoque cualitativo que tenía como objetivo verificar el conocimiento del equipo escolar de una escuela primaria municipal ubicada en la ciudad de Bauru, en el estado de Sao Paulo. La Escuela fue seleccionada de acuerdo con el criterio de tener al menos 1 estudiante con diabetes mellitus tipo 1 (DM1) inscrito en su unidad escolar. Se aplicó un cuestionario que contenía 19 preguntas abiertas y cerradas, que abarcaba el perfil del grupo y los conocimientos sobre la enfermedad. Los resultados mostraron que el $11 \%$ definió correctamente la enfermedad, el 79\% la relacionó por la ausencia de producción de insulina, el $11 \%$ declaró que el control de la glucosa en sangre en la escuela debía realizarse con frecuencia y el $58 \%$ respondió que no vivían juntos con niños DMI en el entorno escolar. Con la realización del estudio, se puede concluir que es indispensable educar al equipo escolar con respecto al conocimiento y la formación en DM1, cuyos esfuerzos conjuntos culminan en el mantenimiento de la calidad de vida del niño matriculado en dicha unidad Escuela.

\footnotetext{
1 Faculdade de Agudos (FAAG), Agudos - SP - Brasil. Pós-graduação em Neuropsicologia. ORCID: https://orcid.org/0000-0002-0221-7321.E-mail: linamarychiti@gmail.com

${ }^{2}$ Universidade Estadual Paulista (UNESP), Bauru - São Paulo - Brasil. Doutor em Educação e Docente do Programa de Mestrado em Docência para a Educação Básica na UNESP/Bauru. ORCID: https://orcid.org/00000003-4957-0058. E-mail: prof.darielcarvalho@gmail.com
} 
PALABRAS CLAVE: Diabetes mellitus tipo 1. Diabetes em escolares. Escuela y salud.

ABSTRACT: Sectional, descriptive study with a qualitative approach that aimed to verify the knowledge of the school team of a municipal elementary school I located in the city of Bauru, in the state of São Paulo. The School was selected according to the criterion of having at least 1 student with type 1 diabetes mellitus (DM1) enrolled in its school unit. A questionnaire containing 19 open and closed questions was applied, covering the profile of the group and the knowledge about the disease. The results showed that $11 \%$ correctly defined the disease, $79 \%$ related it by the absence of insulin production, $11 \%$ stated that blood glucose monitoring at school should be performed frequently and 58\% answered that they did not live together with DM1 children in the school environment. With the accomplishment of the study, it can be concluded that it is indispensable to educate the school team with regard to knowledge and training on DM1, whose joint efforts culminate in the maintenance of the quality of life of the child enrolled in the said unit School.

KEYWORDS: Diabetes mellitus type 1. Diabetes in schoolchildren. School and health.

\section{Introdução}

O Diabetes mellitus (DM) representa um grande desafio para os sistemas de saúde de todo o mundo. De acordo com a Sociedade Brasileira de Diabetes/SBD, (2017) o número de pessoas com diabetes foi projetado para ser superior a 642 milhões em 2040.

Nesse contexto, o Diabetes mellitus tipo 1 (DM1), foco do presente estudo, aparece mundialmente, como uma das principais doenças crônicas da infância (SIMÕES et al, 2009). A International Diabetes Federation/IDF (2015) considera que o Brasil ocupe o terceiro lugar em prevalência de DM1 no mundo com mais de 35 mil pacientes com DM1.

Por definição, o DM1, é uma doença crônica caracterizada pela destruição parcial ou total das células $\beta$ das ilhotas de Langerhans pancreáticas, geralmente causada por processo autoimune (tipo 1 autoimune ou tipo 1A) resultando na incapacidade progressiva da produção de insulina (SBD, 2017). Fator que leva a um quadro de insulinodependência, devido à necessidade de aplicações diárias de insulina para suprir a não produção da mesma.

$\mathrm{Na}$ ausência ou diminuição da insulina ocorre a hiperglicemia (aumento da glicemia) que é o fator primário desencadeador das complicações do DM, como as macroangiopatias (comprometimento das artérias coronarianas, dos membros inferiores e cerebrais) e as microangiopatias que afeta a retina, o glomérulo renal e os nervos (FERREIRA, 2011).

Conforme Góes (2007) ressalta, os principais sintomas decorrentes da hiperglicemia são: polidipsia, poliúria, polifagia e emagrecimento, entretanto sonolência, dores 
generalizadas, formigamentos e dormências, cansaço doloroso nas pernas, câimbras, nervosismo e indisposição podem estar presentes.

Uma das reações adversas mais comuns da doença é a hipoglicemia, ou seja, a redução da glicemia. O Caderno de Atenção Básica $n^{\circ}$ 16, destaca os principais sintomas: sudorese, fadiga, apreensão, tremor, fome, tontura, fraqueza, dor de cabeça, confusão, coma e convulsão que podem ter como causa o excesso de exercício físico, o atraso de uma refeição regular, a pouca ingestão de alimentos e o excesso de insulina (BRASIL, 2006).

Em uma pesquisa de revisão de literatura sobre a correlação da hipoglicemia e o déficit cognitivo, Vida e Bazote (2010) ratificam o dano cerebral estrutural e/ou funcional, causados pelos episódios de hipoglicemia e desencadeadores do déficit cognitivo, sendo essencial o contínuo e intensivo cuidado e educação da criança portadora de DM1 submetida à insulinoterapia, com vistas a reduzir tais comprometimentos.

Fato que requer muita atenção, visto que, Oliveira (2014) alerta que cerca de 90\% dos pacientes que fazem uso da insulinoterapia, já sofreram pelo menos um evento hipoglicêmico, havendo estudos que indicam que a maioria dos doentes sofre uma média dois eventos por semana.

Para alcançar os objetivos do tratamento e do controle metabólico, novos hábitos são fundamentais para o manejo da doença como múltiplas aplicações de insulina diária, automonitorização (testes de glicemia na ponta dos dedos), plano alimentar balanceado e prática regular e planejada de atividades físicas (SOARES; DELL'AGLIO, 2016). "Estar com DM1 significa conviver frequentemente com sintomas decorrentes da hiperglicemia e hipoglicemia" (LA BANCA et al., 2015, p. 9.011).

Esclarecendo que muitos dos sintomas apresentados tanto na hiperglicemia quanto na hipoglicemia podem se relacionar com a aprendizagem devido a letargia para o raciocínio, a fraqueza, ao cansaço, a sonolência, assim como às faltas advindas pelas consultas médicas, como também às possíveis internações (TURATTI, 2012).

Além do mais, conceber uma doença crônica na infância sujeita desordens ao nível biológico, psicológico e cognitivo, devido a dependência de medicações, necessidade de cuidados médicos, psicológicos e educacionais, limitação de suas atividades ou ainda de situações diferenciadas em casa ou na escola (SILVA, 2001).

Sendo assim, o conhecimento das dificuldades e das necessidades da criança com DM1, por parte daqueles que diretamente lidam com ela, é indispensável para que a sua vida decorra com a necessária normalidade (BERNARDO, 2014). 
E é na escola que a criança passa grande parte do seu dia. Muito mais que um espaço destinado à aprendizagem, é também, um espaço para a vivência da ética e da alteridade, podendo dispor do cuidado e da proteção que a criança necessita.

O caderno de Atenção Básica no 24 (Brasil, 2009) evidencia que a escola, que tem como missão primordial desenvolver processos de ensino-aprendizagem, desempenha papel fundamental na formação e atuação das pessoas em todas as arenas da vida social, podendo tornar-se lócus para ações de promoção da saúde para crianças, adolescentes e jovens adultos.

Com tal finalidade, Bomfim et al (2011) elaboraram uma cartilha intitulada: "O aluno com Diabetes: como contribuir para atender às necessidades do aluno com Diabetes" em uma parceria entre os membros das instituições da Faculdade de Medicina de Marília (FANEMA) e da Universidade Estadual Paulista (UNESP) de Marília/SP, que enfatiza a necessidade da colaboração de toda equipe escolar a fim de apoiar o aluno para a manutenção de sua saúde promovendo condições para o desenvolvimento de todas as suas capacidades no ambiente escolar da mesma forma que os demais alunos.

Deste modo, a criança DM1 necessita da parceria entre a família e as áreas da educação e da saúde no ambiente escolar, a fim de lhe garantir segurança e tranquilidade diante de tantas adaptações e dificuldades enfrentadas em seu dia-a-dia. Santana e Silva (2009, p. 670) destacam que “a escola poderá interferir de maneira decisiva na vida de crianças e adolescentes com DM1".

Considerando as necessidades especiais da criança com DM1 e a exigência de esforços conjuntos para que ocorra a manutenção da qualidade de vida da criança diabética, surge o seguinte questionamento: o que a equipe escolar conhece a respeito do DM1 dos alunos que frequentam o seu espaço?

Para tanto, o objetivo de estudo foi de verificar os conhecimentos da equipe escolar a respeito do DMI em escolares de uma escola municipal de Ensino Fundamental I localizada na cidade de Bauru no interior do estado de São Paulo. Por conseguinte, foi eleita uma escola que já tivesse, ao menos, um aluno com DM1 em seu ambiente.

\section{Percurso metodológico}

Trata-se de um estudo seccional, descritivo, com abordagem qualitativa realizado com a equipe escolar de uma instituição pública municipal de educação básica (Fundamental I) do município de Bauru, no Estado de São Paulo, referência regional nas áreas de saúde e de educação. 
Por ser uma pesquisa de campo, oportunizou a vivência dos fatos pesquisados no local em que se desenvolvem. "O estudo de campo apresenta algumas vantagens em relação principalmente aos levantamentos. Como é desenvolvido no próprio local em que ocorrem os fenômenos, seus resultados costumam ser mais fidedignos". (GIL, 2002, p. 56).

A população do estudo foi constituída pelos profissionais da instituição que concordaram em participar da pesquisa e assinaram o Termo de Consentimento Livre e Esclarecido, conforme consta em apêndice, permitindo a coleta de dados e o uso de suas informações.

Foi assegurada a privacidade e o anonimato dos participantes da pesquisa, de modo a proteger suas imagens, bem como garantir que não serão utilizadas as informações coletadas em prejuízo dessas pessoas e/ou da instituição.

A coleta de dados foi realizada mediante aplicação de um questionário composto por 19 questões abertas e fechadas, dividido em duas partes, contemplando:

a) perfil do grupo: cargo, tempo de trabalho no ambiente escolar, idade, sexo, escolarização e contato prévio com crianças DM1 (escola, círculo de amigos, família);

b) abordagem sobre os conhecimentos da equipe escolar sobre DM1 (conceitos, caracterização, causas, sinais e sintomas).

Todas as questões possuíam apenas uma alternativa correta $(\mathrm{C})$, sendo as demais incorretas (E) e uma alternativa alegando desconhecimento da informação (DI). Os respondentes deveriam optar por apenas uma das alternativas.

Para o acesso ao ambiente escolar, previamente foi feito contato com a direção da escola municipal de Ensino Fundamental I, informando sobre a intencionalidade da realização do projeto de pesquisa, devido à presença de uma criança com DM1 no espaço escolar. Após, foi enviada uma cópia do pré-projeto para avaliação da Secretaria Municipal de Educação (SME) de Bauru, evidenciando os objetivos da pesquisa, bem como sua relevância para a comunidade escolar.

Mediante a aprovação da SME, houve novo contato com a direção da escola, que se posicionou favorável à implementação do projeto.

O questionário da pesquisa foi aplicado na escola previamente definida, uma vez que o objetivo da pesquisa era analisar os conhecimentos da equipe escolar sobre DM1 que já tivesse disposto em seu espaço, ao menos um aluno com DM1.

Os procedimentos de aplicação seguiram a premissa da direção da escola que solicitou a aplicação do questionário. Foram entregues 32 questionários, 32 termos de consentimentos 
livres e esclarecidos assinados pela pesquisadora e 32 cópias do termo constando o telefone do pesquisador para que pudessem tirar eventuais dúvidas sobre o projeto de pesquisa.

Após um mês, foram devolvidos 19 questionários e os respectivos termos de consentimentos assinados. Não foi permitido acesso a informações adicionais (livros, internet, amigos) durante o preenchimento do questionário.

\section{Resultados e discussões}

A amostra contemplou 19 profissionais com faixa etária predominante de 41 a 50 anos (53\%), sendo na totalidade (100\%) do sexo feminino. A escolaridade mais apontada foi a do Ensino Superior com Especializações relacionadas a área da Educação (47\%), contrapondo-se aos 26\%, que possuem apenas o Ensino Médio (Tabela 1).

Tabela 1 - Distribuição da amostra por sexo, faixa etária e escolaridade.

\begin{tabular}{c|c|c|c|c|c|c|c|c|c}
\hline Instituição & \multicolumn{3}{|c|}{ Sexo } & \multicolumn{4}{c|}{ Faixa etária } & \multicolumn{4}{c}{ Escolaridade } \\
\hline Municipal & F & M & $31-40$ & $41-50$ & $>50$ & EM & ES & Esp. & M \\
(E. Fund. I) & $100 \%$ & 0 & $16 \%$ & $53 \%$ & $31 \%$ & $26 \%$ & $16 \%$ & $47 \%$ & $11 \%$ \\
\hline
\end{tabular}

Fonte: Elaborado pelos autores.

Os pesquisados foram subdivididos em três grupos:

a) Gestão: 1 diretora e 1 coordenadora;

b) Professoras: 11;

c) Apoio: 2 merendeiras, 1 inspetora e 3 serventes (Tabela 2).

Tabela 2 - Agrupamento da amostra.

\begin{tabular}{c|c|c|c|c|c}
\hline \multicolumn{2}{c|}{ Gestão } & Professoras & \multicolumn{3}{c}{ Apoio } \\
\hline Diretora & Coordenadora & & Merendeiras & Inspetora & Serventes \\
\hline $1(5 \%)$ & $1(5 \%)$ & $11(58 \%)$ & $2(11 \%)$ & $1(5 \%)$ & $3(16 \%)$ \\
\hline
\end{tabular}

Fonte: Elaborado pelos autores.

No que se refere ao tempo de trabalho efetivo no ambiente escolar, evidenciou-se que $42 \%$ dos participantes exercem a função na escola de $04-10$ anos, 37\% de $11-20$ anos e $21 \%$ de 21 - 30 anos, contudo apenas $42 \%$ tiveram contato com crianças DM1 no ambiente escolar: $100 \%$ gestão, $18 \%$ professores e $67 \%$ a equipe de apoio. 
Corroborando com os dados encontrados, Simões et al. (2010) constataram numa pesquisa sobre o conhecimento dos professores sobre o manejo da criança com DM que apenas $15,21 \%$ dos professores responderam afirmativamente quando questionados sobre a existência de comunicação pela direção da escola quanto à presença de alunos matriculados com diagnóstico médico de DM. Essa informação reforça a necessidade de divulgação para toda a comunidade escolar no que se refere a manejos e informações sobre DM, tendo em vista que em algum momento poderá ser necessário o contato ou a colaboração de membros da equipe escolar.

No entanto, é essencial que a criança se sinta amparada e protegida quando distante do cuidador direto (família). "Se bem estruturada e com profissionais bem informados em relação à doença, a escola poderá interferir de maneira decisiva na vida de crianças e adolescentes com DM tipo 1" (SANTANA; SILVA, 2009, p. 670).

A análise do conhecimento da equipe escolar sobre o DM1 demonstrou que apenas $11 \%$ dos participantes ( 2 do grupo de apoio) definiram corretamente a doença. Os demais a relacionaram à obesidade, ao consumo exagerado de açúcares e gorduras, a características familiares ou afirmaram desconhecer a informação.

Todavia, 79\% (2 gestão, 9 professoras e 4 apoio) alegaram que o DM1 se caracteriza pela ausência/diminuição relacionada a produção da insulina, ratificando a compreensão do uso contínuo de insulina pelo acerto de 79\% (2 gestão, 9 professoras e 4 apoio) no questionamento a respeito da substância utilizada em injeções, canetas ou bomba de infusão insulina.

Esses dados mostram que mesmo não tendo uma resposta assertiva com relação a definição do DM1, o relaciona ao termo insulina. Efeito que pode ter sido facilitado pelo uso frequente do termo insulinodependência, usado em larga escala ao paciente diabético, num passado recente. A classificação atual do DM baseia-se na etiologia e não no tipo de tratamento, portanto, os termos "DM insulinodependente" e "DM insulinoindependente" devem ser eliminados dessa categoria classificatória" (SBD, 2017).

Tais informações nos permitem acreditar no conhecimento baseado no senso comum com relação a definição do diabetes num contexto amplo, no qual as pessoas adquiram saberes, por vezes, informações incompletas e/ou improcedentes cientificamente que podem levar a atitudes equivocadas sobre o manejo com o aluno DM1.

Evidenciada por suores, tremores, dor de cabeça, fraqueza e confusão mental, dentre outros, a hipoglicemia pode ser desencadeada pelo excesso na administração de insulina, pela baixa ingesta de carboidratos ou pelo excesso na prática de atividade física (BRASIL, 2006). 
No que concerne a essas questões, ocorreram 32\% de erros (1 gestão, 4 professoras e 2 apoio) relacionados à definição, 58\% (6 professoras e 5 apoio) aos sintomas e 84\% (2 gestão, 9 professoras e 5 apoio) às causas.

A hiperglicemia (aumento da concentração de glicose no sangue) pode ser constatada por diversos sintomas como sonolência, dores generalizadas, formigamentos e dormências, cansaço doloroso nas pernas, câimbras, nervosismo e indisposição (GÓES, 2007) que podem ser ocasionados pela falta de insulina, excesso de carboidratos na alimentação, estresse de uma doença, dentre outros (ICDRS, 2011).

Referindo essas características à pesquisa, constatou-se que ocorreram 53\% de erros (1 gestão, 7 professoras e 2 apoio) com relação a definição, 89\% (1 gestão, 10 professoras e 6 apoio) aos sintomas e $69 \%$ ( 9 professoras e 4 apoio) as causas.

Pôde-se constatar que o maior índice de acertos se relacionou a definição de ambas (hipoglicemia e hiperglicemia), porém há de se considerar que os termos hipo e hiper são habitualmente utilizados para designar escassez e excesso respectivamente (HOUAISS, 2001) em várias outras denominações. Já com relação aos sintomas e as causas foi possível verificar que muitas respostas foram ambíguas, ou seja, elegeram a mesma alternativa para designar ambos eventos.

Contudo, tanto a hipoglicemia quanto a hiperglicemia apresentam causas e sintomas característicos que se identificados a tempo podem ser facilmente resolvidos, sendo imperioso o conhecimento por parte daqueles que convivem por horas com o aluno diabético tipo 1 no ambiente escolar acompanhando e identificando alterações em sua aparência física e em seu comportamento. Simões et al (2009), afirmam que professores devem estar capacitados para reconhecer sintomas e agir conforme a necessidade, possibilitando que problemas futuros sejam evitados.

Outro fator a se considerar é que alguns dos sintomas apresentados na hiperglicemia e na hipoglicemia podem se relacionar com a aprendizagem, tais como: a dificuldade para raciocinar, fraqueza, cansaço, sonolência, visão dupla, dor de cabeça, entre outros (TURATTI, 2012). Não obstante, muitas vezes o professor não sabe identificar o que o aluno apresenta, e por conseguinte, agir nesse momento irrefutável.

Em uma pesquisa realizada no ambulatório de Pediatria da Faculdade de Medicina de Marília/SP, numa parceria entre FAMEMA e UNESP/Marília, com 41 pacientes e 37 pais/responsáveis, Bonfim et al. (2011), constataram que os principais aspectos relacionados à escola foram: episódios de hiperglicemia e hipoglicemia, referidos como o principal problema 
escolar, alimentação escolar não adequada, falta de informação pelos profissionais da escola e a não participação em aulas de educação física, passeios e viagens.

Sendo, portanto, a monitorização da glicemia por meio do capilar sanguíneo a principal ferramenta para a confirmação dos sintomas da hipoglicemia e da hiperglicemia e mais, imprescindível na batalha contra as consequências advindas de tais eventos. "Nesses indivíduos, o uso do método promove a redução do risco de hipoglicemias e amplia a compreensão sobre o efeito dos diversos alimentos, do estresse, das emoções e dos exercícios sobre a glicemia". (SBD, 2017, p. 70).

Apesar disso, somente $11 \%$ dos pesquisados (2 professoras) afirmaram que a monitorização da glicemia na escola deveria ser realizada de maneira frequente. Neste contexto, torna-se indispensável, a necessidade de formação e informação atualizada de toda a equipe escolar, levando-se em conta um aspecto fundamental do tratamento do DM1, a educação.

Um aspecto favorável foi que $95 \%$ dos participantes consideraram importante ter acesso a informações e/ou treinamento sobre DM1 em escolares, independentemente do cargo que ocupam na escola, considerando que todas (100\%) nunca participaram de nenhum tipo de curso/treinamento sobre DM1 em escolares.

\section{Considerações finais}

À criança e ao adolescente são atribuídos direitos: à vida, à alimentação, à educação, à saúde, dentre outros, sendo a escola o espaço em que, cada vez mais, passam um grande tempo do dia e por vários anos. À criança com DM1, esses direitos precisam ser considerados, visto que se trata de uma doença, cuja evolução depende dos cuidados constantes e adequados.

As crianças com DM1 já enfrentam muitas inseguranças no seu cotidiano e dificuldades, por vezes dolorosas, como várias aplicações de insulinas diárias, monitorização da glicemia por meio dos capilares sanguíneos dos dedos das mãos e sintomas desagradáveis advindos das crises de hipoglicemia e de hiperglicemia, como também constantes consultas médicas e exames frequentes de sangue, restrições/substituições de determinados alimentos de acordo com as suas especificidades e na escola necessita sentir-se seguro e amparado diante de tantas adaptações.

Essa pesquisa se ateve a demonstrar os conhecimentos da equipe escolar (diretora, coordenadora, professores, serventes e merendeiras) sobre o DM1 em crianças, evidenciando 
assim, as dificuldades que apresentam em identificar e compreender as necessidades dos escolares com esta patologia. No entanto, devido ao grande tempo com que convivem com a criança, poderiam ser capazes de observar mudanças em seu comportamento e aparência, de forma a reconhecer sinais e sintomas característicos e agir de acordo com a necessidade.

Receber um aluno com DM1, acarreta a necessidade de conhecimento sobre e manejo, características, sintomas e causas de hipoglicemia e de hiperglicemias, monitorização de glicemia e insulinas para tais eventos.

O ideal seria que tivéssemos políticas públicas educativas para a equipe escolar e a presença de um profissional de saúde capacitado (enfermeiro, por exemplo) para lidar adequadamente com as necessidades dos alunos com DM1, bem como de outras patologias crônicas, que acometem as crianças brasileiras no ambiente escolar.

Longe disso, a realidade nos permite acreditar na sinergia entre família e equipe escolar, cujos esforços conjuntos, culminem na manutenção da qualidade de vida da criança.

Acreditamos ser a educação uma alternativa viável para superar a desinformação e o despreparo constatado na equipe escolar sobre DM1. O cuidado somente será aplicado de forma efetiva pela equipe escolar se houver conhecimento e capacitação sobre DM1.

Ressaltamos ainda que este estudo pode ser ampliado com vistas à elaboração de um programa educativo visando o manejo do DM1.

\section{REFERÊNCIAS}

BERNARDO, B. J. R. Conhecimentos dos profissionais da comunidade educativa sobre a diabetes mellitus tipo 1 na criança. Orientadores: João Carvalho Duarte e Maria Isabel Bica Carvalho Costa. 2014, 100 f. Dissertação (mestrado em enfermagem saúde infantil e pediátrica) - escola superior de saúde de Viseu, Portugal. Disponível em: https://repositorio.ipv.pt/bitstream/10400.19/2534/1/BERNARDO\%2C\%20Bruno\%20Jose\%2 0Varandas\%20Ramos\%20-\%20DissertMestrado.pdf. Acesso em: jan. 2020.

BRASIL. Ministério da Saúde. Secretaria de Atenção à Saúde. Departamento de Atenção Básica. Diabetes Mellitus, Brasília: Ministério da Saúde, 2006.

BRASIL. Ministério da Saúde. Secretaria de Atenção à Saúde. Saúde na escola, Brasília: Ministério da Saúde, 2009.

BOMFIM, et al. O aluno com diabetes: como contribuir para atender as necessidades do aluno com diabetes. FAMEMA/UNESP, 1. ed. Marilia, 2011.

FERREIRA, L. T., et al. Diabetes melito: hiperglicemia crônica e suas complicações. Arquivos Brasileiros de Ciências da Saúde, v. 36, n. 3, p. 182-8, set./dez. 2011. 
GIL, A. C. Como elaborar projetos de pesquisa. 4. ed. São Paulo: Atlas, 2002.

GÓES, et al. Diabetes Mellitus Tipo 1 no Contexto Familiar e Social. Rev. Paulista de Ped., Sociedade de Pediatria de São Paulo, v. 25, n. 2, p. 124-128, jun. 2007.

HOUAISS, A. Minidicionário da Língua Portuguesa. 4. ed. Rio de Janeiro: Objetiva, 2010.

INSTITUTO DA CRIANÇA COM DIABETES do RS (ICDRS) - Esclarecimento pais e educadores. Disponível em: http://www.icdrs.org.br/esclarecimentos.php. Acesso em: 31 jul. 2019.

INTERNATIONAL DIABETES FEDERATION. Diabetes Atlas. 7. ed. Brussels, Belgium: International Diabetes Federation; 2015. Disponível em: http://www. diabetesatlas.org/resources/2015-atlas.html. Acesso em: 17 jul. 2019.

LA BANCA, et al. A vivência da criança escolar com diabetes mellitus expressa por meio do brinquedo terapêutico dramático. Rev enferm UFPE on line, Recife, v. 9, n. supl. 7, p. 900917, ago. 2015.

OLIVEIRA, A. M. P. Diabetes mellitus e hipoglicemia. Coimbra, 2014.

SANTANA, E. A; SILVA, S. A.P. Educação física escolar para alunos com diabetes mellitus tipo 1. Motriz, Rio Claro, v. 15, n. 3, p. 669-676, jul./set. 2009.

SILVA, M. G. N. Doenças crônicas na infância: conceito, prevalência e repercussões emocionais. Rev. Pediatria, Ceará, v. 2, n. 2, p. 29-32, maio/ago. 2001.

SIMÕES et. al. Interface pesquisa-extensão na capacitação de professores para o manejo da criança diabética. $I n$ : $61^{\circ}$ CONGRESSO BRASILEIRO DE ENFERMAGEM, 2009, Fortaleza.

SIMÕES et al. Conhecimento dos professores sobre o manejo da criança com diabetes mellitus. Rev. Enferm, Florianópolis, v. 19, n. 4, p. 651-7, out./dez. 2010.

SOARES, J. P. G.; DELL'AGLIO, D. Relações entre qualidade de vida e diabetes mellitus tipo 1 na adolescência. 2016 unisinos - doc: 10.4013/ctc 2016.92.02. Contextos clínicos, v. 9, n. 2, jul./dez. 2016.

SOCIEDADE BRASILEIRA DE DIABETES (SBD). Diretrizes da Sociedade Brasileira de Diabetes. São Paulo: Clannad, 2017-2018.

TOURATTI, C. R. Educação e saúde: um olhar sobre direitos e cuidados do aluno com diabetes mellitus tipo 1. 2011. 154 f. Dissertação (Mestrado em Educação) - Universidade do Sul de Santa Catarina, Tubarão, 2011.

VIDA, A.; BAZOTTE, R. B. Hipoglicemia induzida por insulina como fator desencadeador de déficit cognitivo em crianças portadoras de Diabetes Mellitus tipo 1. Arq. Ciênc. Saúde UNIPAR, Umuarama, v. 14, n. 2, p. 153-156, maio/ago. 2010. 


\section{Como referenciar este artigo}

CAMARGO, Linamary Chiti; CARVALHO, Dariel. Conhecimentos da equipe escolar sobre diabetes mellitus tipo 1 em escolares. Revista Ibero-Americana de Estudos em Educação, Araraquara, v. 15, n. 2, p. 619-630, abr./jun. 2020. e-ISSN: 1982-5587. DOI: https://doi.org/10.21723/riaee.v15i2.13327

Submetido em: 16/02/2019

Revisões requeridas: $30 / 06 / 2019$

Aprovado em: 20/12/2019

Publicado em: 20/02/2020 